\title{
Case influence diagnostics for the significance of the linear regression model
}

\author{
Whasoo Bae ${ }^{a}$, Soyoung $\mathrm{Noh}^{b}$, Choongrak Kim${ }^{1, b}$ \\ ${ }^{a}$ Department of Statistics, Inje University, Korea; \\ ${ }^{b}$ Department of Statistics, Pusan National University, Korea
}

\begin{abstract}
In this paper we propose influence measures for two basic goodness-of-fit statistics, the coefficient of determination $R^{2}$ and test statistic $F$ in the linear regression model using the deletion method. Some useful lemmas are provided. We also express the influence measures in terms of basic building blocks such as residual, leverage, and deviation that showed them as increasing function of residuals and a decreasing function of deviation. Further, the proposed measure reduces computational burden from $O(n)$ to $O(1)$. As illustrative examples, we applied the proposed measures to the stackloss data sets. We verified that deletion of one or few influential observations may result in big change in $R^{2}$ and $F$-statistic.
\end{abstract}

Keywords: coefficient of determination, $F$-statistic, goodness-of-fit, influential observations

\section{Introduction}

Research on regression diagnostics were initiated by Cook (1977) that have continued to most existing statistical models with a few exceptions. Good reviews on representative results in linear regression diagnostics are Belsley et al. (1980), Chatterjee and Hadi (1986), and Cook and Weisberg (1982). Diagnostic results in Box-Cox transformation model (Box and Cox, 1964) have also been done by Cook and Wang (1983), Hinkley and Wang (1988), Kim et al. (1996), and Tsai and Wu (1990). Walker and Birch (1988) derived a version of Cook's distance in the ridge regression (Hoerl and Kennard, 1970), and Kim et al. (2015) proposed a type of Cook's distance in the lasso regression model.

Studies on regression diagnostics have also been done in regards to nonparametric regression. In the spline smoothing model, Eubank (1985), Kim (1996), and Silverman (1985) suggested versions of Cook's distance, and Kim et al. (2001) suggested Cook's distance in the local polynomial regression, and Fung et al. (2002) and Kim et al. (2002) studied detection of influential observations in the semiparametric model. Bae et al. (2008) also studied diagnostic issues in the varying coefficient model.

In this paper, we study diagnostic issues in goodness-of-fit measures in the linear regression model. We develop influence measures for goodness-of-fit statistics such as the $F$-statistic and the coefficient of determination $R^{2}$. Many of the suggested influence measures in many statistical models are concerned with detecting influential observations on estimators of regression coefficients. As far as we know, influence measures for goodness-of-fit statistics have not yet been studied. By using the deletion

\footnotetext{
${ }^{1}$ Corresponding author: Department of Statistics, Pusan National University, 2, Busandaehak-ro 63 beon-gil, Geumjeonggu, Busan 46241, Korea. E-mail: crkim@pusan.ac.kr
}

Published 31 March 2017 / journal homepage: http://csam.or.kr

(C) 2017 The Korean Statistical Society, and Korean International Statistical Society. All rights reserved. 
method, we also express the proposed influence measures for goodness-of-fit statistics in terms of basic building blocks such as residuals and deviations. This paper is organized as follows. In Section 2, influence measures for goodness-of-fit statistics are suggested and analytic expression for goodnessof-fit statistics in terms of basic building blocks are given. Illustrative examples based on real data sets are given in Section 3, and concluding remarks are given in Section 4.

\section{Influence measures for goodness-of-fit statistics}

\subsection{Basic notations}

Consider the linear regression model

$$
\boldsymbol{y}=\boldsymbol{X} \boldsymbol{\beta}+\boldsymbol{\epsilon},
$$

where $\boldsymbol{y}$ is an $n$-vector of responses, $\boldsymbol{X}$ is an $n \times p$ full column matrix of known covariates, $\boldsymbol{\beta}$ is a $p$ vector of unknown coefficients, and $\epsilon$ is an $n$-vector of independent Gaussian random variables with mean zero and unknown variance $\sigma^{2}$. We use $y_{i}$ and $\boldsymbol{x}_{i}$ to denote the $i^{\text {th }}$ row of $\boldsymbol{y}$ and $\boldsymbol{X}$, respectively, and use the subscript $(K)$ to indicate the deletion of $k$ observations in an index set $K=\left\{i_{1}, i_{2}, \ldots, i_{k}\right\}$. Therefore, $\boldsymbol{X}_{(i)}$ denotes the matrix $\boldsymbol{X}$ with the $i^{\text {th }}$ row deleted. However, the subscript $K$ indicates the corresponding $k$ observations in an index set $K$. Therefore, $\boldsymbol{X}_{K}$ denotes the $k \times p$ submatrix of $\boldsymbol{X}$. After fitting the model by the method of least squares, we have $\hat{\boldsymbol{\beta}}=\left(\boldsymbol{X}^{\prime} \boldsymbol{X}\right)^{-1} \boldsymbol{X}^{\prime} \boldsymbol{y}$, and $\hat{\boldsymbol{y}}=\boldsymbol{H} \boldsymbol{y}$, where $\boldsymbol{H}=\boldsymbol{X}\left(\boldsymbol{X}^{\prime} \boldsymbol{X}\right)^{-1} \mathbf{X}^{\prime}$ is the hat matrix. Let residual vector be $\boldsymbol{e}=\boldsymbol{y}-\hat{\boldsymbol{y}}$ and $s^{2}=\boldsymbol{e}^{\prime} \boldsymbol{e} /(n-p)$ be the unbiased estimator of $\sigma^{2}$.

Here, we consider the influence of $k$ observations on the coefficient of determination $R^{2}$ and the $F$-test statistic. Recall that

$$
R^{2}=\frac{\mathrm{SST}-\mathrm{SSE}}{\mathrm{SST}},
$$

where $\operatorname{SST}=\sum\left(y_{i}-\bar{y}\right)^{2}$ is the total sum of squares (SST), and SSE $=\sum\left(y_{i}-\hat{y}_{i}\right)^{2}$ is the error sum of squares (SSE). Also, the $F$-test statistic is given by

$$
F=\frac{\mathrm{SST}-\mathrm{SSE}}{(p-1) s^{2}}
$$

Therefore, both statistics, $R^{2}$ and $F$, are functions of SST, SSE, and $s^{2}$.

\subsection{Useful Lemmas}

Let $R_{(K)}^{2}$ and $F_{(K)}$ be the coefficient of determination and the $F$-statistic, respectively, based on $(n-k)$ observations after deleting $k$ observations in a set $K$. Now, to detect influential observations on $R^{2}$ and $F$, we suggest influence measures as: Define $\Delta D_{K}=R^{2}-R_{(K)}^{2}$ and $\Delta F_{K}=F-F_{(K)}$ as influence measures for the coefficient of determination and the $F$-statistic, respectively. Hence, they can be rewritten as

$$
\Delta D_{K}=\frac{\operatorname{SSE}_{(K)}}{\operatorname{SST}_{(K)}}-\frac{\mathrm{SSE}}{\mathrm{SST}}
$$

and

$$
\Delta F_{K}=\frac{(n-p)(\mathrm{SST}-\mathrm{SSE})}{(p-1) \mathrm{SSE}}-\frac{(n-p-k)\left(\mathrm{SST}_{(K)}-\mathrm{SSE}_{(K)}\right)}{(p-1) \mathrm{SSE}_{(K)}}
$$


where $\operatorname{SST}_{(K)}$ and $\mathrm{SSE}_{(K)}$ denote the SST and the SSE, respectively, based on $(n-k)$ observations after deleting $k$ observations in a set $K$.

Lemma 1. $S S T_{(K)}=S S T-1 /(n-k)\left(\sum_{j \in K} d_{j}\right)^{2}-\sum_{j \in K} d_{j}^{2}$, where $d_{j}=y_{j}-\bar{y}$.

Proof: Let $\bar{y}_{K}=(1 / k) \sum_{j \in K} y_{j}$ and $\bar{y}_{(K)}=(1 /(n-k)) \sum_{j \notin K} y_{j}$. Now,

$$
\begin{aligned}
\operatorname{SST}_{(K)} & =\sum_{j \notin K}\left(y_{j}-\bar{y}_{(K)}\right)^{2} \\
& =\sum_{j=1}^{n}\left(y_{j}-\bar{y}_{(K)}\right)^{2}-\sum_{j \in K}\left(y_{j}-\bar{y}_{(K)}\right)^{2} .
\end{aligned}
$$

Since $\sum_{j=1}^{n}\left(y_{j}-\bar{y}\right)=0$ and $\bar{y}-\bar{y}_{(K)}=(1 /(n-k)) \sum_{j \in K} d_{j}$, we have

$$
\begin{aligned}
\operatorname{SST}_{(K)} & =\operatorname{SST}+\frac{n}{(n-k)^{2}}\left(\sum_{j \in K} d_{j}\right)^{2}-\sum_{j \in K} d_{j}^{2}-\frac{2}{n-k}\left(\sum_{j \in K} d_{j}\right)^{2}-\frac{k}{(n-k)^{2}}\left(\sum_{j \in K} d_{j}\right)^{2} \\
& =\mathrm{SST}-\frac{1}{n-k}\left(\sum_{j \in K} d_{j}\right)^{2}-\sum_{j \in K} d_{j}^{2} .
\end{aligned}
$$

Lemma 2. $S S E_{(K)}=s^{2}\left(n-p-r_{K}^{2}\right)$, where $r_{K}^{2}=\boldsymbol{e}_{K}{ }^{\prime}\left(\boldsymbol{I}-\boldsymbol{H}_{K}\right)^{-1} \boldsymbol{e}_{K} / s^{2}, \boldsymbol{e}_{K}=\left(e_{i_{1}}, \ldots, e_{i_{k}}\right)^{\prime}$, and $\boldsymbol{H}_{K}=\boldsymbol{X}_{K}\left(\boldsymbol{X}^{T} \boldsymbol{X}\right)^{-1} \boldsymbol{X}_{K}$.

Proof: First, we note that

$$
\begin{aligned}
\operatorname{SSE}_{(K)} & =\sum_{j \notin K}\left(y_{j}-\mathbf{x}_{j}^{\prime} \hat{\boldsymbol{\beta}}_{(K)}\right)^{2} \\
& =\left(\boldsymbol{y}-\boldsymbol{X} \hat{\boldsymbol{\beta}}_{(K)}\right)^{\prime}\left(\boldsymbol{y}-\boldsymbol{X} \hat{\boldsymbol{\beta}}_{(K)}\right)-\left(\boldsymbol{y}_{K}-\boldsymbol{X}_{K} \hat{\boldsymbol{\beta}}_{(K)}\right)^{\prime}\left(\boldsymbol{y}_{K}-\boldsymbol{X}_{K} \hat{\boldsymbol{\beta}}_{(K)}\right) .
\end{aligned}
$$

Also, recall that

$$
\hat{\boldsymbol{\beta}}-\hat{\boldsymbol{\beta}}_{(K)}=\left(\boldsymbol{X}^{\prime} \boldsymbol{X}\right)^{-1} \boldsymbol{X}_{K}^{\prime}\left(\boldsymbol{I}-\boldsymbol{H}_{K}\right)^{-1} \boldsymbol{e}_{K},
$$

therefore, we have

$$
\boldsymbol{y}-\boldsymbol{X} \hat{\boldsymbol{\beta}}_{(K)}=\boldsymbol{e}+\boldsymbol{X}\left(\boldsymbol{X}^{\prime} \boldsymbol{X}\right)^{-1} \boldsymbol{X}_{K}^{\prime}\left(\boldsymbol{I}-\boldsymbol{H}_{K}\right)^{-1} \boldsymbol{e}_{K},
$$

and

$$
\boldsymbol{y}_{K}-\boldsymbol{X}_{K} \hat{\boldsymbol{\beta}}_{(K)}=\boldsymbol{e}_{K}+\boldsymbol{H}_{K}\left(\boldsymbol{I}-\boldsymbol{H}_{K}\right)^{-1} \boldsymbol{e}_{K} .
$$

Finally, we have

$$
\operatorname{SSE}_{(K)}=\mathrm{SSE}+\boldsymbol{e}_{K}^{\prime}\left\{-\boldsymbol{I}-\left(\boldsymbol{I}-\boldsymbol{H}_{K}\right)^{-1} \boldsymbol{H}_{K}\right\} \boldsymbol{e}_{K},
$$


and note that

$$
\boldsymbol{I}+\left(\boldsymbol{I}-\boldsymbol{H}_{K}\right)^{-1} \boldsymbol{H}_{K}=\left(\boldsymbol{I}-\boldsymbol{H}_{K}\right)^{-1} .
$$

Then,

$$
\begin{aligned}
\operatorname{SSE}_{(K)} & =\operatorname{SSE}\left(1-\frac{r_{K}^{2}}{n-p}\right) \\
& =s^{2}\left(n-p-r_{K}^{2}\right)
\end{aligned}
$$

which completes the proof of Lemma 2.

\subsection{Influence measures}

By Lemmas 1 and 2, we have

$$
\begin{aligned}
\Delta D_{K} & =R^{2}-R_{(K)}^{2} \\
& =\frac{\operatorname{SSE}\left(1-\frac{r_{K}^{2}}{n-p}\right)}{\mathrm{SST}-\frac{1}{n-k}\left(\sum_{j \in K} d_{j}\right)^{2}-\sum_{j \in K} d_{j}^{2}}-\frac{\mathrm{SSE}}{\mathrm{SST}},
\end{aligned}
$$

and

$$
\begin{aligned}
\Delta F_{K} & =F-F_{(K)} \\
& =\frac{\frac{\operatorname{SST}-\mathrm{SSE}}{p-1}}{\frac{\mathrm{SSE}}{n-p}}-\frac{(n-p-k)\left(\mathrm{SST}-\frac{1}{n-k}\left(\sum_{j \in K} d_{j}\right)^{2}-\sum_{j \in K} d_{j}^{2}-\operatorname{SSE}\left(1-\frac{r_{K}^{2}}{n-p}\right)\right)}{(p-1)\left(\operatorname{SSE}\left(1-\frac{r_{K}^{2}}{n-p}\right)\right)} .
\end{aligned}
$$

As a special case, we consider deleting a single observation, the $i^{\text {th }}$ observation, say. Then, we can easily express them as: For notational simplicity, let

$$
q_{i}=\frac{d_{i}^{2}}{\mathrm{SST}}
$$

be the proportion of the $i^{t h}$ deviation, and $r_{i}=e_{i} /\left(s \sqrt{1-h_{i i}}\right)$ be the $i^{\text {th }}$ internally standardized residual, and $r_{i}^{*}=e_{i} /\left(s_{(i)} \sqrt{1-h_{i i}}\right)$ be the $i^{\text {th }}$ externally standardized residual. Then, we have

$$
\begin{aligned}
\Delta D_{i} & =\frac{\operatorname{SSE}_{(i)}}{\mathrm{SST}_{(i)}}-\frac{\mathrm{SSE}}{\mathrm{SST}} \\
& =\left(1-R^{2}\right) \cdot \frac{\left(\frac{n}{n-1} q_{i}-\frac{r_{i}^{2}}{n-p}\right)}{1-\frac{n}{n-1} q_{i}} .
\end{aligned}
$$

Also, for the influence of the $i^{t h}$ observation on $F$, we have

$$
\begin{aligned}
\Delta F_{i} & \equiv F-F_{(i)} \\
& =F \cdot\left\{1-\frac{1-\left(\frac{n}{n-1} d_{i}^{2}-r_{i}^{2} s^{2}\right) / \mathrm{SSR}}{r_{i}^{2} / r_{i}^{* 2}}\right\} .
\end{aligned}
$$


Table 1: Values of $\Delta D_{i}, \Delta F_{i}$ with the standardized residual $r_{i}$, the leverage $h_{i i}$, and the proportion of deviation $q_{i}$ in the stackloss data

\begin{tabular}{|c|c|c|c|c|c|c|c|c|c|}
\hline \multicolumn{2}{|c|}{$\Delta D_{i}$} & \multicolumn{2}{|c|}{$\Delta F_{i}$} & \multicolumn{2}{|c|}{$r_{i}$} & \multicolumn{2}{|c|}{$h_{i i}$} & \multicolumn{2}{|c|}{$q_{i}$} \\
\hline Obs. & Value & Obs. & Value & Obs. & Value & Obs. & Value & Obs. & Value \\
\hline 21 & -0.035 & 21 & -38.9 & 21 & -2.638 & 17 & 0.412 & 1 & 0.290 \\
\hline 1 & 0.027 & 1 & 18.4 & 4 & 1.882 & 2 & 0.318 & 2 & 0.183 \\
\hline 2 & 0.017 & 2 & 13.9 & 3 & 1.546 & 1 & 0.302 & 3 & 0.183 \\
\hline 4 & -0.014 & 4 & -8.4 & 1 & 1.193 & 21 & 0.285 & 16 & 0.054 \\
\hline 3 & 0.006 & 3 & 7.2 & 9 & -1.046 & 7 & 0.219 & 4 & 0.053 \\
\hline 6 & -0.005 & 16 & 6.7 & 12 & 0.969 & 8 & 0.219 & 15 & 0.044 \\
\hline 9 & -0.005 & 18 & 6.3 & 6 & -0.965 & 12 & 0.217 & 17 & 0.044 \\
\hline 16 & 0.005 & 19 & 5.7 & 11 & 0.884 & 14 & 0.206 & 18 & 0.044 \\
\hline 12 & -0.004 & 17 & 5.0 & 7 & -0.834 & 10 & 0.200 & 19 & 0.035 \\
\hline 18 & 0.004 & 14 & 4.5 & 15 & 0.809 & 15 & 0.190 & 13 & 0.021 \\
\hline 7 & -0.003 & 13 & 4.0 & 2 & -0.716 & 3 & 0.175 & 14 & 0.015 \\
\hline 11 & -0.003 & 15 & 4.0 & 17 & -0.611 & 19 & 0.175 & 12 & 0.010 \\
\hline 19 & 0.003 & 10 & 3.2 & 5 & -0.542 & 18 & 0.161 & 10 & 0.006 \\
\hline 17 & 0.002 & 20 & 3.0 & 8 & -0.485 & 13 & 0.158 & 11 & 0.006 \\
\hline 5 & -0.001 & 8 & 2.9 & 13 & -0.480 & 11 & 0.155 & 8 & 0.003 \\
\hline 8 & -0.001 & 5 & 2.4 & 20 & 0.454 & 9 & 0.140 & 9 & 0.003 \\
\hline 13 & 0.001 & 7 & 1.0 & 10 & 0.437 & 16 & 0.131 & 20 & 0.003 \\
\hline 14 & 0.001 & 11 & 1.0 & 16 & 0.299 & 4 & 0.129 & 21 & 0.003 \\
\hline 15 & 0.001 & 12 & 0.6 & 19 & -0.203 & 20 & 0.080 & 7 & 0.001 \\
\hline 20 & -0.001 & 9 & -0.5 & 18 & -0.153 & 6 & 0.077 & 5 & 0.000 \\
\hline 10 & 0.000 & 6 & 0.0 & 14 & -0.017 & 5 & 0.052 & 6 & 0.000 \\
\hline
\end{tabular}

All the statistics are given in a descending order in absolute values. Obs. = observations.

Based on $\Delta D_{i}$ and $\Delta F_{i}$, we see that $r_{i}^{2}$ and $q_{i}$ play key roles in determining influential observations on $R^{2}$ and $F$. Both $R^{2}$ and $F$ increase as we delete $i^{\text {th }}$ observation with large residual $r_{i}^{2}$; however, they decrease as we delete $i^{\text {th }}$ observation with large $q_{i}$. This result is quite interesting in the sense that the Cook's distance is an increasing function of $h_{i i}$, but both $R^{2}$ and $F$ are a decreasing function of $q_{i}$. Recall that $q_{i}$ can be regraded as the $i^{\text {th }}$ leverage of the response, while $h_{i i}$ is the leverage of covariates.

Remark 1. In regression diagnostics, "Influential on what?" is a really important question for a particular influence measure. For example, the Cook's distance (Cook, 1977) is an influence measure to see the effect of observations on the (scaled) change of the estimates of regression coefficients. Therefore, influential observations detected by the Cook's distance may not be influential to other estimators such as the change of variance estimate or the $F$-statistic. Two influence measures, $\Delta D_{i}$ and $\Delta F_{i}$, proposed in this paper are related to the significance of the postulated model. Therefore, $\Delta D_{i}$ deals with the influence on the change of the proportion of regression sum of squares among the SST. However, $\Delta F_{i}$ is concerned about the change of the $F$-statistic when testing $H_{0}: \boldsymbol{\beta}=\mathbf{0}$. Hence, influential observations based on $\Delta D_{i}$ may not be influential based on $\Delta F_{i}$, and vice versa.

\section{Numerical study}

As an illustrative example for the proposed influence measures, we consider the stackloss data, consisting of one response variable $Y$ and three explanatory variables $X_{1}, X_{2}, X_{3}$ with 21 observations. After fitting the response on three covariates using the linear model, we obtained that $R^{2}=0.9136$ and $F=59.9$.

Now, we compute the proposed influence measures $\Delta D_{i}, \Delta F_{i}$ with the standardized residual $r_{i}$, the leverage $h_{i i}$, and the proportion of deviation $q_{i}$ in the stackloss data in Table 1. Table 2 also summarizes the five largest values of $\Delta D_{K}$ and $\Delta F_{K}$ when $k=1,2,3$. From both tables, we note the following: 
Table 2: Five largest values (in absolute) of $\Delta D_{K}$ and $\Delta F_{K}$ when $k=1,2,3$ in the stackloss data

\begin{tabular}{ccccc}
\hline \hline$k$ & $K$ & $\Delta D_{K}$ & $K$ & $\Delta F_{K}$ \\
\hline & 21 & -0.035 & 21 & -38.921 \\
1 & 1 & 0.027 & 1 & 18.357 \\
& 2 & 0.017 & 2 & 13.853 \\
& 4 & -0.014 & 4 & -8.373 \\
& 3 & 0.006 & 3 & 7.249 \\
\hline \multirow{2}{*}{2} & 1,2 & 0.079 & 4,21 & -96.900 \\
& 4,21 & -0.056 & 6,21 & -40.427 \\
& 1,3 & 0.039 & 13,21 & -39.813 \\
& 6,21 & -0.039 & 9,21 & -37.202 \\
& 13,21 & -0.039 & 5,21 & -35.925 \\
\hline & $1,2,3$ & 0.153 & $4,13,21$ & -121.462 \\
& $1,2,16$ & 0.091 & $4,6,21$ & -100.135 \\
& $1,2,18$ & 0.088 & $3,4,21$ & -99.731 \\
\hline
\end{tabular}

From $\Delta D_{i}$ and $\Delta F_{i}$, we see that four observations $\{21\},\{1\},\{2\},\{4\}$ seem to be influential on $R^{2}$ and $F$. They also have large values of $r_{i}, h_{i i}$, and $q_{i}$. Among 4 influential observations, deletion of $\{21\}$ and $\{4\}$ increases $R^{2}$ and $F$, however, deletion of $\{1\}$ and $\{2\}$ decreases $R^{2}$ and $F$. This phenomenon occurs when $k=2$, i.e., influential sets are $\{1,2\}$ and $\{4,21\}$. If we delete $\{4,21\}$, then $R^{2}$ increases from 0.9136 to 0.9696 , and $F$ increases from 59.9 to 156.8 .

We should also note that both $R^{2}$ and $F$ increase as we delete $i^{\text {th }}$ observation with large residual $r_{i}^{2}$; however, they decrease as we delete $i^{\text {th }}$ observation with large $q_{i}$, i.e., when we delete $\{21\}$, observation with large residual $r_{i}$, both $R^{2}$ and $F$ increase, however, they decrease as we delete observations either $\{1\},\{2\}$, or $\{3\}$. This phenomenon occurs when we delete multiple cases of observations (Table 2).

Table 2 also indicates that observation $\{21\}$ is very influential on both the coefficient of determination and the $F$-statistic; therefore, when we delete observation $\{21\}$ the $F$-statistic increases from 59.9 to 98.8. We now evaluate $\Delta t_{i}=t-t_{(i)}$ for each covariate to see how the $t$-statistic for each covariate change as we delete one observation (Table 3). Here, we see that when we delete observation $\{21\}$, the $t$-statistic for $X_{1}$ increases from 5.307 to 7.481, while the $t$-statistic for $X_{2}$ decreases from 3.520 to 2.513. Therefore, when we find influential observations on the $F$-statistic, it is necessary to find which covariate is seriously affected by the influential observations.

\section{Concluding remarks}

Most of diagnostic issues are studied about regression models. In this paper, we proposed influence measures for two basic goodness-of-fit statistics, the coefficient of determination $R^{2}$ and the overall significance test statistic $F$. We have derived analytic formula for two measures and expressed them as basic building blocks such as residuals, leverages and deviations. From the analytic expressions, we found that both $R^{2}$ and $F$ increase as we delete $i^{t h}$ observation with large residual $r_{i}^{2}$; however, they decrease as we delete $i^{\text {th }}$ observation with large $q_{i}$. We also applied them to the real data set and verified that deletion of one or few influential observations may result in big change in $R^{2}$ and $F$.

It would be useful for further research to study the cutoff or threshold for the proposed influence measures. We also expect that the bootstrap method will be one other possible method. 
Table 3: Values of $\Delta t_{i}=t-t_{(i)}$, change in the $t$-statistic when the $i^{t h}$ observation is deleted, in the stackloss data

\begin{tabular}{|c|c|c|c|c|c|c|c|}
\hline \multicolumn{2}{|c|}{$X_{0} \equiv 1$} & \multicolumn{2}{|c|}{$X_{1}$} & \multicolumn{2}{|c|}{$X_{2}$} & \multicolumn{2}{|c|}{$X_{3}$} \\
\hline Obs. & Value & Obs. & Value & Obs. & Value & Obs. & Value \\
\hline 21 & 1.249 & 21 & -2.174 & 21 & 1.007 & 7 & 0.052 \\
\hline 17 & -1.115 & 4 & -0.771 & 4 & 0.438 & 8 & -0.109 \\
\hline 13 & -0.294 & 1 & 0.581 & 13 & 0.364 & 1 & -0.048 \\
\hline 18 & -0.292 & 7 & 0.562 & 3 & -0.168 & 10 & -0.130 \\
\hline 19 & -0.241 & 8 & 0.522 & 14 & 0.164 & 15 & -0.185 \\
\hline 14 & -0.227 & 9 & 0.494 & 9 & -0.162 & 17 & -1.115 \\
\hline 15 & -0.185 & 10 & 0.454 & 19 & 0.135 & 11 & -0.100 \\
\hline 4 & 0.175 & 12 & 0.383 & 10 & 0.113 & 2 & -0.037 \\
\hline 10 & -0.130 & 19 & 0.373 & 6 & -0.106 & 12 & -0.018 \\
\hline 8 & -0.109 & 3 & 0.288 & 18 & 0.103 & 4 & 0.175 \\
\hline 11 & -0.100 & 18 & 0.236 & 8 & 0.100 & 21 & 1.249 \\
\hline 16 & -0.092 & 11 & 0.182 & 20 & 0.090 & 14 & -0.227 \\
\hline 9 & 0.078 & 16 & 0.177 & 16 & 0.088 & 20 & -0.056 \\
\hline 5 & -0.061 & 13 & 0.163 & 12 & -0.085 & 9 & 0.078 \\
\hline 20 & -0.056 & 14 & 0.163 & 11 & -0.081 & 13 & -0.294 \\
\hline 3 & -0.055 & 6 & 0.148 & 1 & 0.068 & 5 & -0.061 \\
\hline 7 & 0.052 & 2 & 0.144 & 7 & -0.053 & 18 & -0.292 \\
\hline 1 & -0.048 & 5 & 0.127 & 5 & 0.051 & 6 & 0.029 \\
\hline 2 & -0.037 & 17 & 0.124 & 17 & 0.023 & 19 & -0.241 \\
\hline 6 & 0.029 & 20 & 0.118 & 15 & 0.011 & 16 & -0.092 \\
\hline 12 & -0.018 & 15 & 0.016 & 2 & 0.005 & 3 & -0.055 \\
\hline
\end{tabular}

Obs. = observations.

\section{Acknowledgement}

This work was supported by the 2016 Inje University Research Grant.

\section{References}

Bae W, Hwang S, and Kim C (2008). Influence diagnostics in the varying coefficient model with longitudinal data, Computational Statistics, 23, 185-196.

Belsley DA, Kuh E, and Welsch RE (1980). Regression Diagnostics: Identifying Influential Data and Sources of Collinearity, Wiley, New York.

Box GEP and Cox DR (1964). An analysis of transformations, Journal of the Royal Statistical Society Series B (Methodological), 26, 211-252.

Chatterjee S and Hadi AS (1986). Influential observations, high leverage points, and outliers in linear regression, Statistical Science, 1, 379-393.

Cook RD (1977). Detection of influential observation in linear regression, Technometrics, 19, 15-18.

Cook RD and Wang PC (1983). Transformations and influential case in regression, Technometrics, 25, 337-343.

Cook RD and Weisberg S (1982). Characterizations of an empirical influence function for detecting influential cases in regression, Technometrics, 22, 495-508.

Eubank RL (1985). Diagnostics for smoothing splines, Journal of the Royal Statistical Society Series $B$ (Methodological), 47, 332-341.

Fung WK, Zhu ZY, Wei BC, and He X (2002). Influence diagnostics and outlier tests for semiparametric mixed models, Journal of the Royal Statistical Society Series B (Statistical Methodology), 64, 565-579.

Hinkley DV and Wang S (1988). More about transformations and influential cases in regression, 
Technometrics, 30, 435-440.

Hoerl AE and Kennard RW (1970). Ridge regression: biased estimation for nonorthogonal problems, Technometrics, 12, 55-67.

Kim C (1996). Local influence and replacement measure, Communications in Statistics - Theory and Methods, 25, 49-61.

Kim C, Storer BE, and Jeong M (1996). Note on Box-Cox transformation diagnostics, Technometrics, 38, 178-180.

Kim C, Lee Y, and Park BU (2001). Cook's distance in local polynomial regression, Statistics and Probability Letters, 54, 33-40.

Kim C, Park BU, and Kim W (2002). Influence diagnostics in semiparametric regression models, Statistics and Probability Letters, 60, 49-58.

Kim C, Lee J, Yang H, and Bae W (2015). Case influence diagnostics in the lasso regression, Journal of the Korean Statistical Society, 44, 271-279.

Silverman BW (1985). Density Estimation for Statistics and Data Analysis, Chapman and Hall, London.

Tsai CL and Wu X (1990). Diagnostics in transformation and weighted regression, Technometrics, 32, $315-322$.

Walker E and Birch JB (1988). Influence measures in ridge regression, Technometrics, 30, 221-227.

Received December 16, 2016; Revised January 18, 2017; Accepted January 20, 2017 